\title{
High-throughput transcriptomic analysis reveals new players in popcorn (Zea mays) aluminum resistance
}

Vitor Batista Pinto ( $\square$ vitorbts@yahoo.com.br)

Universidade Federal de Vicosa https://orcid.org/0000-0002-8119-7268

Priscila Gonçalves Ferreira

Universidade Federal de Vicosa

Pedro Marcus Pereira Vidigal

Universidade Federal de Vicosa

Tiago Antônio de Oliveira Mendes

Universidade Federal de Vicosa

Maximiller Dal-Bianco

Universidade Federal de Vicosa

José Marcelo Soriano Viana

Universidade Federal de Vicosa

Research article

Keywords: Zea mays

Posted Date: April 23rd, 2020

DOI: https://doi.org/10.21203/rs.2.15209/v2

License: (c) (1) This work is licensed under a Creative Commons Attribution 4.0 International License.

Read Full License 
The authors have withdrawn this preprint from Research Square 